\title{
DLA systems in GRB afterglows
}

\section{P. M. Vreeswijk ${ }^{1}$, S. L. Ellison ${ }^{2}$, C. Ledoux ${ }^{1}$, R. A. M. J. Wijers ${ }^{3}$, J. P. U. Fynbo ${ }^{4}$, P. Møller ${ }^{5}$, and J. Hjorth ${ }^{4}$}

${ }^{1}$ European Southern Observatory, Alonso de Cordova 3107, Casilla 19001, Santiago 19, Chile

${ }^{2}$ University of Victoria, 3800 Finnerty Rd, Victoria, BC, V8P 1A1, Canada

${ }^{3}$ Astronomical Institute 'Anton Pannekoek', University of Amsterdam \& Center for High

Energy Astrophysics, Kruislaan 403, 1098 SJ Amsterdam, The Netherlands

${ }^{4}$ Niels Bohr Institute, University of Copenhagen, Juliane Maries Vej 30, DK-2100 Copenhagen $\varnothing$, Denmark

${ }^{5}$ European Southern Observatory, Karl-Schwarzschild-Strasse 2, D-85748, Garching bei München, Germany

\begin{abstract}
To date, half a dozen DLAs have been detected in GRB afterglows, but most have modest signal-to-noise ratios. Our detection of a DLA in the afterglow of GRB 030323 is unambiguous: its neutral hydrogen column density is $\log \mathrm{N}(\mathrm{HI})=21.90 \pm 0.07$, higher than any (GRBor QSO-) DLA HI column density inferred directly from Ly $\alpha$ in absorption. We discuss several other properties such as the metallicity, the detection of fine-structure lines of SiII, and our strong upper limit on the $\mathrm{H}_{2}$ content. The GRB afterglow allows us to infer all these quantities, even with the host being as faint as $\mathrm{V}=28$. The successful launch of the Swift satellite (November 2004) has made high-resolution spectroscopy of a large sample of GRB afterglows possible. This will offer a unique way to study in detail the interstellar medium inside GRB host galaxies as a function of redshift.
\end{abstract}

\section{Introduction}

Damped Ly $\alpha$ (DLA) systems are conventionally found in absorption toward QSOs; they are characterised by their neutral hydrogen column density: $\log \mathrm{N}(\mathrm{HI}) \geqslant 20.3$. The HI column density distribution of QSO absorption-line systems, ranging from the lowdensity Ly $\alpha$ forest, through the Lyman limit systems to the higher-density DLA systems, follows a power law with index of about -1.5 , which means that the bulk of the neutral hydrogen in the Universe is contained within the highest Hi column density systems: the DLAs. Despite intense searches, only a few counterparts of DLA absorbers have been detected (see Møller et al. 2002b); the persistent light of the background QSO makes deep imaging of the DLAs very difficult. Linking DLAs with galaxy type has therefore proven to be difficult; some advocate large, fast-rotating, thick-disk galaxies (e.g. Wolfe et al. 1995), others prefer faint, gas-rich dwarfs (Haehnelt et al. 1998; Ledoux et al. 1998).

\section{GRB-DLAs}

Figure 1 shows that gamma-ray bursts (GRBs) are, just as QSOs, distant and bright sources. Therefore, they can be used in exactly the same way as QSOs have been used for decades to enable detailed study of the interstellar medium (ISM) of any absorption system on the line-of-sight. However, there are clear differences between these two probes. The first difference is that GRBs do not alter their environment in such a drastic way as QSOs. This allows study of the GRB host-galaxy ISM, which is unperturbed beyond a radius of several tens of parsec from the GRB explosion site. Moreover, a handful of GRBs have been found to be associated with supernova explosions (Galama et al. 1998; 

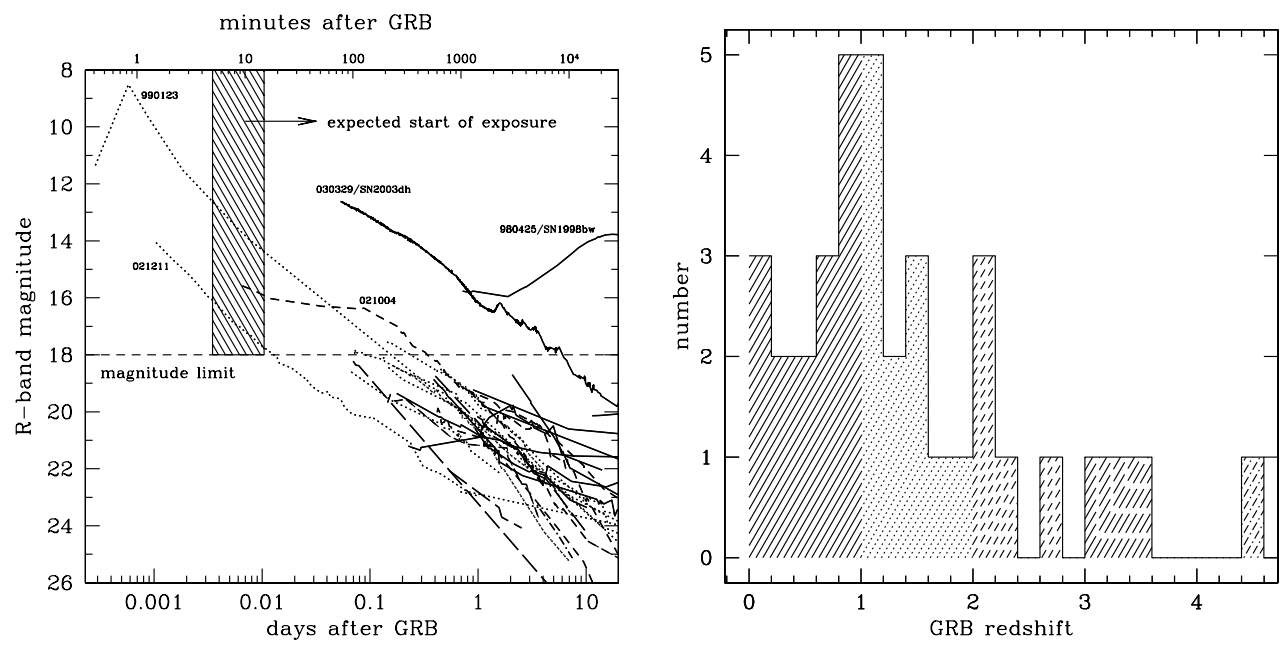

Figure 1. On the left: R-band light curves of all pre-Swift afterglows with a measured redshift. The line type of the curves correspond to the line type of the hashed regions in the redshift histogram on the right (e.g. solid light curves correspond to GRBs between $z=0$ and $z=1$ ). Swift has made prompt $(<15$ min.) activation possible, when some fraction of the afterglows will be bright enough for high-resolution spectroscopy.

Hjorth et al. 2003b), which suggests that at least some GRBs are caused by the collapse of a massive star. Hence, the GRB sight-line probably leads us directly to regions of massive-star formation. And third, a QSO sticks around for some years to come, but a GRB afterglow fades away extremely rapidly (see Fig. 1). This makes it very difficult to obtain a high signal-to-noise high-resolution spectrum. To date very few GRB spectra, if any, are comparable to the quality of the typical QSO spectra. But the advantage of this is that once the afterglow has faded, it leaves a clear view of the sight-line. This would allow foreground lower-z absorbers, if any are detected in the spectrum, to be seen in emission more easily than in QSO lines of sight.

In several GRB spectra, evidence for the presence of a DLA line has been found (Jensen et al. 2001; Fynbo et al. 2001; Hjorth et al. 2003a; Jakobsson et al. 2004). In this talk we will focus on the DLA discovered in a low-resolution spectrum $(\mathrm{R} \sim 2,000)$ of GRB 030323 at $z=3.372$, and use it as an example of what can be done in the near future for a larger sample and at a higher resolution.

\section{The high column-density DLA in GRB 030323}

Following the HETE-II detection of GRB 030323 (Graziani et al. 2003) and the discovery of its optical counterpart by Gilmore et al. (2003), we performed follow-up spectroscopic observations. The combined, de-reddened VLT/FORS2 spectrum (with a resolution $\mathrm{R} \sim 2,000$ ) of GRB 030323 is shown in Fig. 2. We identify many metal absorption lines from different ions: Nv, SiI, SiII, Siı**, Sirv, Oı, Ciı*, Ciı, CIv, FeII, and Alıı, strong Ly $\alpha$ absorption, and the intervening $\operatorname{Ly} \alpha$ forest blue-ward of this. All the identified lines are indicated in Fig. 2. The average redshift of the metal absorption lines is $z=3.372 \pm 0.001$.

A fit to the strong Ly $\alpha$ absorption line yields $\log \mathrm{N}(\mathrm{HI})=21.90 \pm 0.07$, which is the highest column density measured so far for a (QSO- or GRB-) DLA system. Fig. 3 shows a comparison of the HI column density distribution of QSO-DLAs (taken from the compilation of Curran et al. 2002) and pre-Swift GRB-DLAs (Jensen et al. 2001; Fynbo 


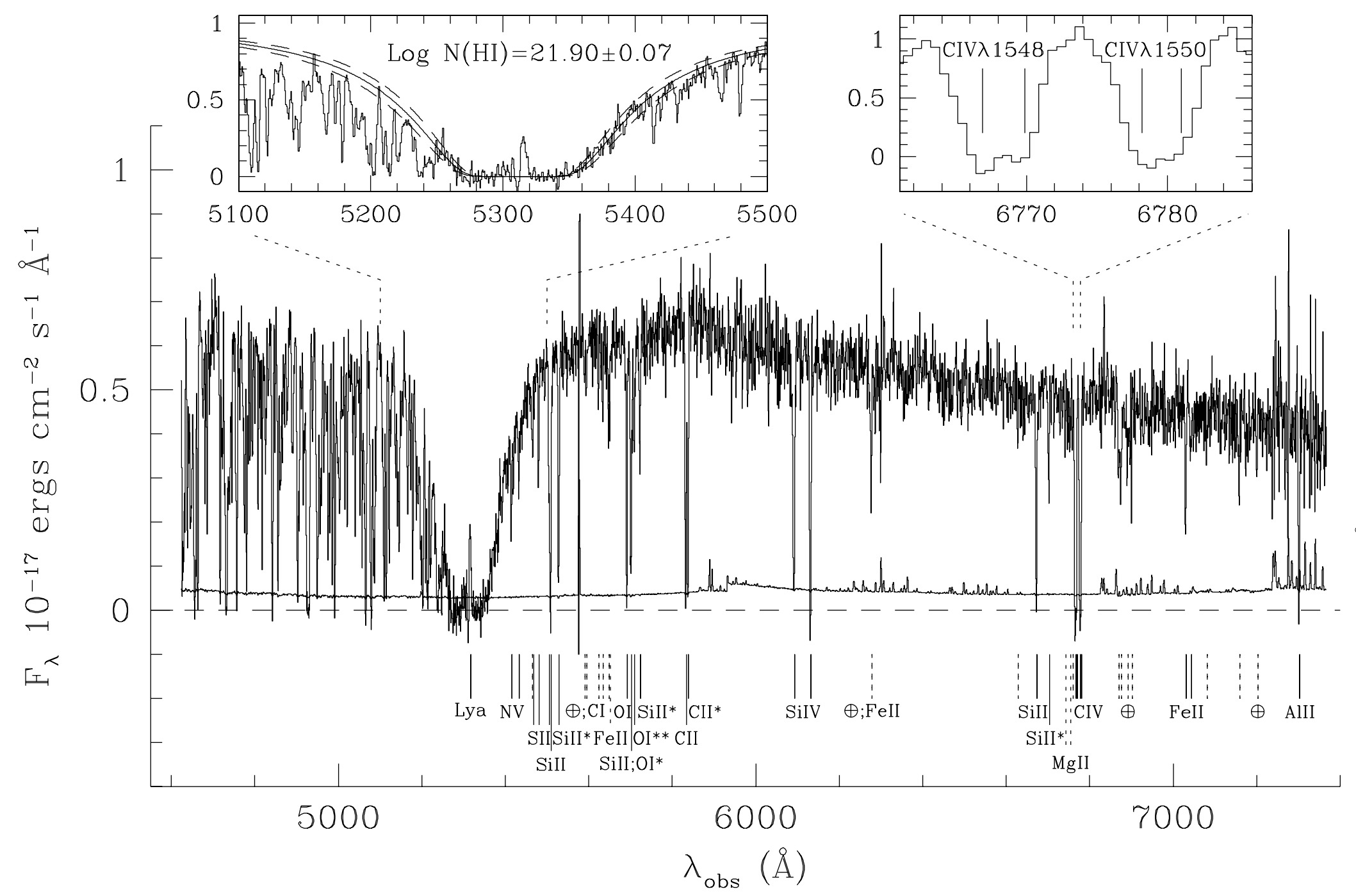

Figure 2. Combined VLT/FORS2 spectrum of GRB 030323, including the Poisson error spectrum. The left inset shows the normalised spectrum with the model fitting to the damped Ly $\alpha$ line, including the $1 \sigma$ errors. This column density is currently the highest of any DLA line measured through Ly $\alpha$ in absorption (see Fig. 3). 
et al. 2001; Hjorth et al. 2003a). For completeness, we also show the GRB for which Ly $\alpha$ was detected but which does not qualify as a DLA: GRB 021004 (Møller et al. 2002a). It is quite striking that out of $7 \mathrm{GRB}$ afterglows for which $\mathrm{Ly} \alpha$ was redshifted into the observable spectrum, 6 show evidence for a DLA.

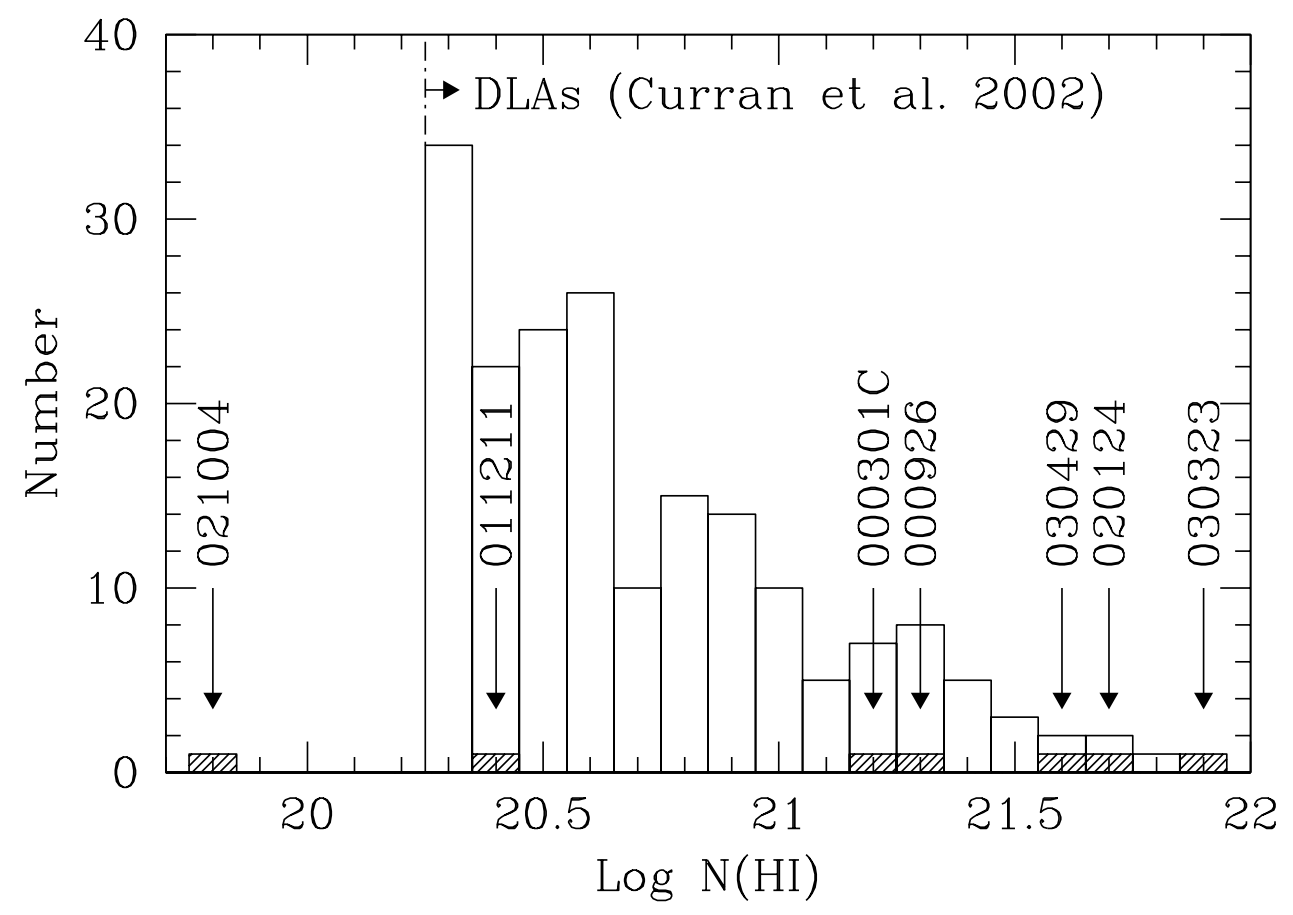

Figure 3. Histogram of the column densities of DLA systems measured through the damping wings of Ly $\alpha$ discovered against a background QSO, taken from Curran et al. (2002). We do not include the sub-DLA systems on this plot, which are located at column densities lower than log $\mathrm{N}(\mathrm{HI})=20.3$; i.e. the apparent cut-off is not real. The shaded histogram shows pre-Swift GRBs for which the redshift was large enough to detect Ly $\alpha$. Out of 7 GRBs, 6 show neutral hydrogen column densities above the DLA threshold $\mathrm{N}(\mathrm{HI}) \geqslant 2 \times 10^{20}$ atoms $\mathrm{cm}^{-2}$.

It is generally assumed that the apparent HI column density limit of $\log \mathrm{N}(\mathrm{HI}) \sim 22$ for QSO-DLAs is due to an observational bias against the detection of such high-column density systems, as these would obscure the background QSO if they contain some dust (e.g. Ostriker \& Heisler 1984; Fall \& Pei 1993). However, a radio-selected QSO survey for DLAs by Ellison et al. (2001) did not uncover a previously unrecognised population of $\mathrm{N}(\mathrm{HI})>10^{21} \mathrm{~cm}^{-2}$ DLAs in front of faint QSOs. An alternative scenario was proposed by Schaye (2001): the lack of high Hi column density systems would be due to the conversion of $\mathrm{HI}$ to $\mathrm{H}_{2}$ molecules as the neutral gas density increases. In GRB 030323, we do not see any evidence for the presence of $\mathrm{H}_{2}$ to support this scenario. Future GRBs with possible larger Hi column densities will provide further constraints on the existence of such a conversion of the neutral gas to $\mathrm{H}_{2}$ at high $\mathrm{HI}$ column densities.

\section{Metallicity, $\mathrm{H}_{2}$ content, and silicon $\mathrm{II}^{*}$ detection}

From the few unsaturated metal lines detected in the GRB 030323 spectrum, we measure iron and sulphur metallicities of $[\mathrm{Fe} / \mathrm{H}]=-1.5 \pm 0.2$ and $[\mathrm{S} / \mathrm{H}]=-1.3 \pm 0.2$. In Fig. 4, 
we compare the GRB-DLAs for which the Zn, S, or Si metallicity has been measured (GRB 000926 and the GRB 030323 measurement above) with a sample of QSO-DLAs taken from Prochaska et al. (2003b). Although the GRB-DLA sample is still small, there is a hint that GRB-DLAs are more metal-rich than QSO-DLAs, which one would expect if GRBs originate in massive-star forming regions. We note that Savaglio and colleagues (Savaglio et al. 2003) also pointed out large Zn column densities in three GRB host galaxies (one of which is GRB 000926) with respect to QSO-DLAs, while they found the Fe column densities to be similar to those of QSO-DLAs.

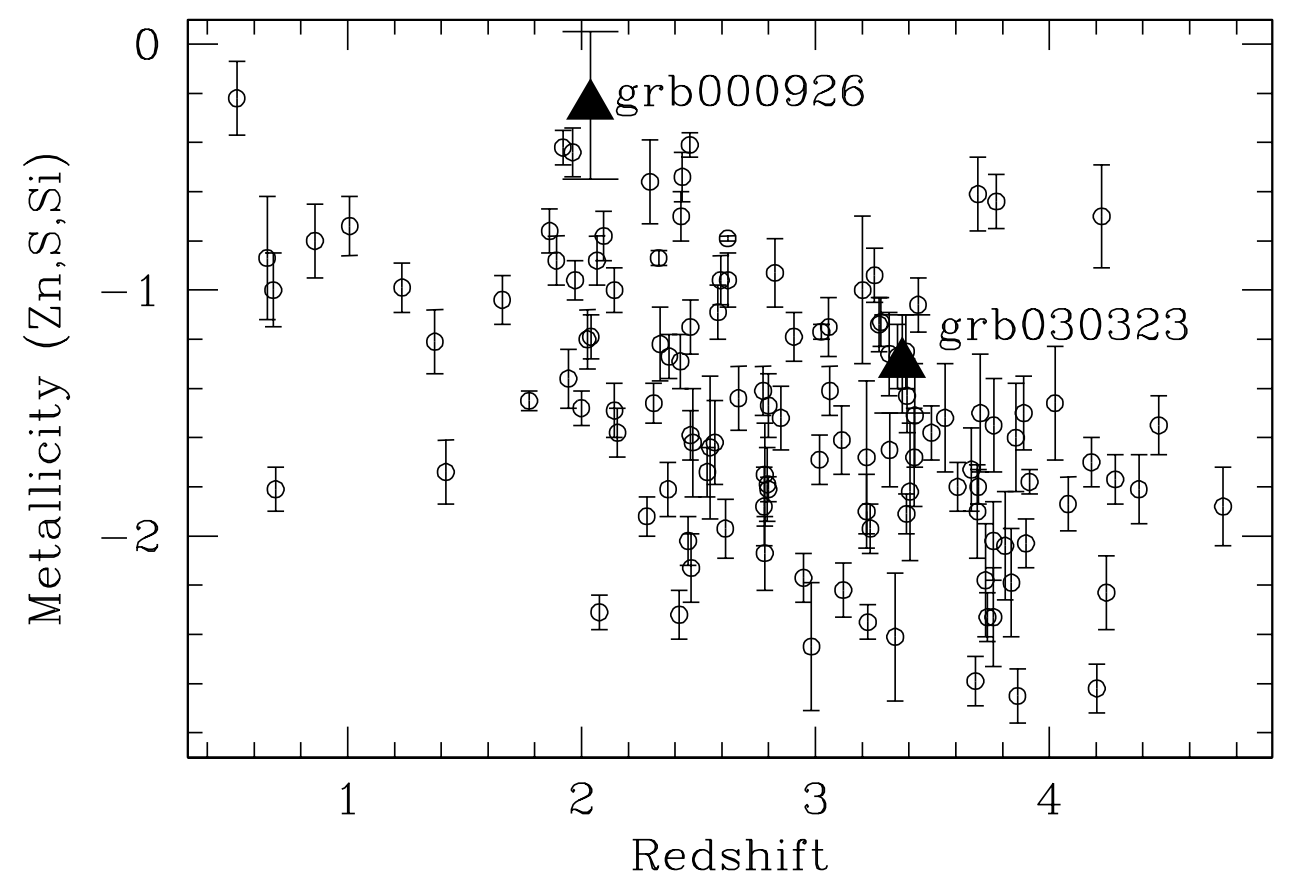

Figure 4. Comparison of the metallicities of a sample of QSO-DLAs, taken from Prochaska et al. (2003a) (open circles), with the two GRBs for which a metallicity has been determined (solid triangles): GRB 000926 and GRB 030323. Although the GRB-DLA sample is too small to draw any conclusions, the GRB hosts seem to be located at the metal-rich end of the QSO-DLA distribution.

From the detection of the fine-structure lines SiII* $\lambda \lambda$ 1309, 1533 (see Fig. 2), we very crudely estimate the particle density: $\mathrm{n}_{\mathrm{H}^{0}} \sim 10^{2-4} \mathrm{~cm}^{-3}$, under the assumption that these fine-structure levels are populated by collisions, and not through direct excitation by infra-red photons (which is not an important excitation mechanism in the case of SiII) or fluorescence (see Silva \& Viegas 2002). This particle density is higher than typically inferred for QSO-DLA environments (Silva \& Viegas 2002). As this line has never been clearly detected up to now in QSO-DLAs, the detection of this and other SirI* lines in the GRB 030323 spectrum suggests an origin in the vicinity of the GRB place of birth (e.g. the star-forming region in which it exploded). From the measured Hi column density and the rough estimate of the particle density, we infer a size of $26 \mathrm{pc}$ for the SiII* absorbing region; as the total Hi column density that we measured may not be all associated with the SiI* absorbing region, this size estimate is actually an upper limit. With the particle density so high, one would expect hydrogen molecules to be 
present, which we do not detect. We obtain a rather strong upper limit on the molecular fraction: $f \equiv 2 N\left(\mathrm{H}_{2}\right) /\left(2 N\left(\mathrm{H}_{2}\right)+N(\mathrm{HI})\right) \leqslant 10^{-6}$, for both the GRB environment and the host galaxy of GRB 030323. This can be explained by the low metallicity of the gas (see Ledoux et al. 2003), but it may also be that the molecules in the vicinity of the GRB explosion have been dissociated by the strong GRB UV/X-ray emission (e.g. Draine \& Hao 2002).

The spectrum of GRB 030323 shown in Fig. 2 was taken when the afterglow brightness was around $R=21.5$. NASA's new GRB satellite, Swift, was successfully launched in November 2004. Although the satellite is still in its calibration phase, the instruments are generally behaving as expected. In the Swift era, the burst alert delay will be shorter, and the error circle will be considerably smaller, allowing afterglows to be discovered when they are brighter. The first results on the optical afterglows of Swift GRBs indicate that Swift is detecting bursts with fainter afterglows than the sample of pre-Swift bursts (Swift is at least five times more sensitive in the $\gamma$-ray regime than previous missions). Still, Swift will allow us to perform high-resolution spectroscopy of a large sample of afterglows, which can provide statistical information about the distribution of the gas in high-redshift star-forming regions, in addition to the evolution of the metallicity, dust, and $\mathrm{H}_{2}$ contents of GRB host galaxies.

\section{References}

Curran, S. J., Webb, J. K., Murphy, M. T., et al., 2002, Publications of the Astronomical Society of Australia, 19, 455

Draine, B. T., Hao, L., 2002, ApJ, 569, 780

Ellison, S. L., Yan, L., Hook, I. M., et al., 2001, A\&A, 379, 393

Fall, S. M., Pei, Y. C., 1993, ApJ, 402, 479

Fynbo, J. P. U., Gorosabel, J., Møller, P., et al., 2001, in Lighthouses of the Universe: The Most Luminous Celestial Objects and Their Use for Cosmology, Proc. of the MPA/ESO/MPE/USM Joint Astronomy Conf., eds. M. Gilfanov, R. Sunyaev \& E. Churazov (Garching: Springer), p. 187; preprint astro-ph/0110603

Galama, T. J., Vreeswijk, P. M., Van Paradijs, J., et al., 1998, Nature, 395, 670

Gilmore, A., Kilmartin, P., Henden, A., 2003, GRB Circular Network, 1949

Graziani, C., Shirasaki, Y., Matsuoka, M., et al., 2003, GRB Circular Network, 1956

Haehnelt, M. G., Steinmetz, M., Rauch, M., 1998, ApJ, 495, 647

Hjorth, J., Møller, P., Gorosabel, J., et al., 2003a, ApJ, 597, 699

Hjorth, J., Sollerman, J., Møller, P., et al., 2003b, Nature, 423, 847

Jakobsson, P., Hjorth, J., Fynbo, J. P. U., et al., 2004, A\&A, 427, 785

Jensen, B. L., Fynbo, J. P. U., Gorosabel, J., et al., 2001, A\&A, 370, 909

Ledoux, C., Petitjean, P., Bergeron, J., Wampler, E. J., Srianand, R., 1998, A\&A, 337, 51

Ledoux, C., Petitjean, P., Srianand, R., 2003, MNRAS, 346, 209

Møller, P., Fynbo, J. P. U., Hjorth, J., et al., 2002a, A\&A, 396, L21

Møller, P., Warren, S. J., Fall, S. M., Fynbo, J. P. U., Jakobsen, P., 2002b, ApJ, 574, 51

Ostriker, J. P., Heisler, J., 1984, ApJ, 278, 1

Prochaska, J. X., Gawiser, E., Wolfe, A. M., Castro, S., Djorgovski, S. G., 2003a, ApJ, 595, L9

Prochaska, J. X., Gawiser, E., Wolfe, A. M., Cooke, J., Gelino, D., 2003b, ApJS, 147, 227

Savaglio, S., Fall, S. M., Fiore, F., 2003, ApJ, 585, 638

Schaye, J., 2001, ApJ, 562, L95

Silva, A. I., Viegas, S. M., 2002, MNRAS, 329, 135

Wolfe, A. M., Lanzetta, K. M., Foltz, C. B., Chaffee, F. H., 1995, ApJ, 454, 698 